\title{
MONOTONE GRADIENTS ON BANACH LATTICES
}

\author{
K. SUNDARESAN
}

Dedicated to Professor Victor L. Klee on his sixtieth birthday

\begin{abstract}
It is well known that a differentiable real valued function on the real line is convex iff its derivative is nondecreasing. This characterization of differentiable convex functions does not extend if the domain of the function is a Banach lattice of dim $\geq 2$. In this paper a complete characterization of a subclass of differentiable convex functions on a given Banach lattice is obtained in terms of the monotonicity and natural orthogonality properties of the gradients.
\end{abstract}

It is well known that a differentiable real valued function on the real line is convex iff its derivative is nondecreasing. It is also known that a $C^{1}$-real valued function $F$ on an arbitrary Banach space $E$ is convex iff the gradient of $F$, say $f$, satisfies the condition $(*)(f(x)-f(y), x-y) \geq 0$ for all $x, y \in E$. Condition (*) is referred to in the literature as a monotonicity condition. However when $E$ is a Banach lattice, it is natural to define a function $f$ on $E$ into another Banach lattice $E_{1}$ as nondecreasing if $f(x) \geq f(y)$ whenever $x, y$ in $E$ are such that $x \geq y$. The present investigation originated out of the surprising fact that, as shown by suitable examples, the characterization of $C^{1}$-convex real valued functions on the real line recalled above, does not extend if the domain of the function is a Banach lattice of $\operatorname{dim} \geq 2$, and the monotonicity of the gradient function is interpreted as in the preceding sentence. More specifically, if $E$ is a Banach lattice of $\operatorname{dim} \geq 2$, there are $C^{1}$-convex real valued functions on $E$ with gradients not nondecreasing and there are $C^{1}$-real valued functions on $E$ with gradients nondecreasing which fail to be convex. The main result here provides a complete characterization of $C^{1}$-convex real valued functions $\Phi$ on an arbitrary Banach lattice $E$ of which the translates $\Phi(x+y)-\Phi(x)$ are orthogonally additive in $y$ for all $x$ in $E$, in terms of the monotonicity and natural orthogonality properties of the gradient map of $\Phi$. The characterization of the special class of $C^{1}$-convex functions on Banach lattices stated in the main result here appears to be a reasonable substitute of the characteristic property of $C^{1}$-convex real valued functions on the real line recalled above.

The plan of the paper is as follows. In the introductory section, $\S 1$, the basic definitions and known results are recalled for convenience of reference, and the main results are stated and proved in $\S 2$.

1. Preliminaries. If $E$ is a Banach space, a continuous mapping $f: E \rightarrow E^{*}$, where $E^{*}$ is the dual of $E$, is called a gradient map if there is a real valued $C^{1}$ function $F$ on $E$ such that $F^{\prime}(x)=f(x)$ for all $x$ in $E$. It is known that if $f$ is a gradient map on $E$, then the real valued function $F$ defined on $E$ by setting $F(x)=\int_{0}^{1} f(t x) x d t$ is a $C^{1}$-real valued function such that $F^{\prime}(x)=f(x)$ for all $x$ in $E$ (see (2.5.6) in Berger [1]). If $E$ and $E_{1}$ are Banach lattices, then a function $f: E \rightarrow E_{1}$ is said to be nondecreasing if $f(x) \geq f(y)$ for all $x, y$ in $E$ such that

Received by the editors July 3, 1985 .

1980 Mathematics Subject Classification (1985 Revision). Primary 26A24, 46B30. 
$x \geq y$. If $E$ is a Banach lattice, and $E_{1}$ is an arbitrary vector space, a function $f: E \rightarrow E_{1}$ is said to be orthogonally additive, in short o.a., if $f(x+y)=f(x)+f(y)$ for all pairs of orthogonal vectors $x, y(x \perp y)$ in $E$, i.e. vectors $x, y$ in $E$ such that $|x| \wedge|y|=0$. A real valued function $f$ on a vector space $E$ is said to be convex if $f(a x+b y) \leq a f(x)+b f(y)$ for all $x, y$ in $E$ and for all nonnegative real numbers $a, b$ such that $a+b=1$. If $f$ is a function on a vector space $E$, the $x$-translate of $f$ for $x$ in $E$ is the function $g$ defined on $E$ by setting $g(y)=f(x+y)-f(x)$.

For an account of differential analysis in Banach spaces, see Berger [1] , Cartan [2], Dieudonné [4], Lang [7], and Sundaresan and Swaminathan [13]. For the theory as well as terminology concerning Banach lattices, see Lacey [6], Lindenstrauss and Tzafriri $[\mathbf{8}]$, and Schaefer [11]. It is to be noted that orthogonally additive functionals on Banach lattices, in particular on function spaces, have been extensively studied by Mizel and his collaborators $[\mathbf{9}, \mathbf{1 0}]$, and Drewnowski and Orlicz [3].

Before concluding this preliminary section a useful result is recalled. A real valued function $\Phi$ on a Banach lattice $E$ is symmetrically monotone if for all $x$ in E

$$
\Phi(x+y)+\Phi(x-y) \leq \Phi\left(x+y^{\prime}\right)+\Phi\left(x-y^{\prime}\right)
$$

if $y, y^{\prime}$ in $E$ satisfy the inequality $|y| \leq\left|y^{\prime}\right|$. A well-known result of Hardy, Littlewood, and Polya [5] asserts that a continuous real valued function on the real line is convex if and only if it is symmetrically monotone. This characterization of convex real valued functions on the real line is false if the domain of the function is a Banach lattice of $\operatorname{dim} \geq 2$. The following result stated and proved in Sundaresan $[\mathbf{1 2}]$ is recalled here for convenience of reference.

THEOREM 1. If $E$ is an arbitrary Banach lattice, then a continuous real valued function $\phi$ on $E$ is symmetrically monotone if and only if it is convex and for each $x$ in $E$, the $x$-translate of $\phi$ defined by $\phi(x+y)-\phi(x)$ is orthogonally additive in $y$.

For a proof, see [12].

2. Main results. Before proceeding to the promised characterization several useful results are stated with proofs.

LEMMA 2. If $\phi$ is a symmetrically monotone real valued function on a Banach lattice $E$, and if $x, y$, and $z$ are in $E$ such that $0 \leq x \leq z$ and $y \geq 0$, then

(a) $\phi(x+y)-\phi(x) \leq \phi(z+y)-\phi(z)$,

(b) $\phi(-x+y)-\phi(-x) \geq \phi(-z+y)-\phi(-z)$.

PROOF. With $x, y$, and $z$ as in the hypothesis, let $\xi=\frac{1}{2}(x+y+z), \eta=$ $\frac{1}{2}(x+y-z)$, and $\eta^{\prime}=\frac{1}{2}(z+y-x)$. Then $|\eta| \leq \frac{1}{2}(z-x)+\frac{1}{2} y=\eta^{\prime}=\left|\eta^{\prime}\right|$. Since $\phi$ is symmetrically monotone

$$
\phi(\xi+\eta)+\phi(\xi-\eta) \leq \phi\left(\xi+\eta^{\prime}\right)+\phi\left(\xi-\eta^{\prime}\right) .
$$

Thus $\phi(x+y)+\phi(z) \leq \phi(z+y)+\phi(x)$, from which (a) follows. Once again choosing

$$
\xi=\frac{-x+y-z}{2}, \quad \eta=\frac{-x+y+z}{2}, \quad \text { and } \quad \eta^{\prime}=\frac{-z+y+x}{2},
$$

proceeding as before, after noting $\left|\eta^{\prime}\right| \leq|\eta|$, inequality (b) is deduced. 
THEOREM 3. If $\phi$ is a symmetrically monotone real valued $C^{1}$-function on a Banach lattice $E$, and the mapping $f: E \rightarrow E^{*}$ is the gradient of $\phi$ then (a) for each $x$ in $E$, the $x$-translate of $f$ is o.a., (b) if $y \perp z$, then $f(y) z=f(0) z$, and more generally $f(x+y) z=f(x) z$ for all $x$ in $E$, and (c) $f$ is a nondecreasing function.

Proof. Let $\xi \in E$, and let $\psi_{\xi}$ be the $\xi$-translate of $\phi$. Since $\phi$ is symmetrically monotone in $E, \psi_{\xi}$ is o.a. by Theorem 1 . Now let $y_{1}, y_{2}$ be two orthogonal vectors in $E$ and $x \in E$. If $h \in E$, for real numbers $t$, from the o.a. property of $\psi_{\xi}$, for $\xi=x+t h$, and $\xi=x$, it follows that

$$
\begin{aligned}
\phi\left(x+y_{1}+y_{2}+t h\right) & -\phi\left(x+y_{1}+y_{2}\right) \\
= & \phi\left(x+y_{1}+t h\right)+\phi\left(x+y_{2}+t h\right)-\phi(x+t h) \\
& -\left[\phi\left(x+y_{1}\right)+\phi\left(x+y_{2}\right)-\phi(x)\right] .
\end{aligned}
$$

Dividing by $t$, and proceeding to the limit as $t \rightarrow 0$, it follows that

$$
f\left(x+y_{1}+y_{2}\right) h=f\left(x+y_{1}\right) h+f\left(x+y_{2}\right) h-f(x) h
$$

for all $h \in E$. Thus the $x$-translate of $f$ is o.a. verifying (a).

Next let $y, z$ be in $E$ such that $y \perp z$. Since $\phi(x)-\phi(0)$ is o.a. in $x$, and $y \perp t z$ for all real $t, \phi(y+t z)-\phi(0)=\phi(y)-\phi(0)+\phi(t z)-\phi(0)$ if $y \perp z$. Thus $\phi(y+t z)-\phi(y)=$ $\phi(t z)-\phi(0)$, from which (b) is deduced. The second conclusion in (b) is deduced from the preceding result applied to the $x$-translate of $\phi$, which is verified to be a $C^{1}$ symmetrically monotone function with gradient at $y$ equal to $f(x+y)$ for all $y$ in $E$.

To verify that $f$ is nondecreasing, let $x, z$ be in $E$ such that $x \leq z$. Thus $x^{+} \leq z^{+}, x^{-} \geq z^{-}$. From Lemma 2 it follows that for $y \geq 0$ and $t \geq 0$,

$$
\phi\left(x^{+}+t y\right)-\phi\left(x^{+}\right) \leq\left(z^{+}+t y\right)-\phi\left(z^{+}\right)
$$

and

$$
\phi\left(-x^{-}+t y\right)-\phi\left(-x^{-}\right) \leq \phi\left(-z^{-}+t y\right)-\phi\left(-z^{-}\right) .
$$

From these inequalities it is deduced that

$$
f\left(x^{+}\right) y \leq f\left(z^{+}\right) y \text { and } f\left(-x^{-}\right) y \leq f\left(-z^{-}\right) y .
$$

Since by part (a), $f-f(0)$ is o.a., it follows that

$$
f(\xi)-f(0)=f\left(\xi^{+}\right)+f\left(-\xi^{-}\right)-2 f(0) \text { for all } \xi \in E .
$$

(1) and (2) together imply that $f(x) y \leq f(z) y$ for all $y \geq 0$, verifying $f(x) \leq f(z)$ if $x \leq z$, as desired.

The converse of the preceding is proved in the next theorem after noting three useful lemmas.

LEMMA 4. If $E$ is a vector lattice and $g: E \rightarrow E_{1}$ is an orthogonally additive map, where $E_{1}$ is a vector space, then

$$
g(x)+g(-x)=g(y)+g(-y)=g(|x|)+g(-|x|) \quad \text { if }|x|=|y| .
$$

PROOF. If suffices to verify that

$$
g(x)+g(-x)=g(|x|)+g(-|x|) .
$$


Let $x=x^{+}-x^{-}$, so that $|x|=x^{+}+x^{-}$. Since $x^{+} \perp x^{-}$

$$
\begin{aligned}
g(x)+g(-x) & =g\left(x^{+}\right)+g\left(-x^{-}\right)+g\left(-x^{+}\right)+g\left(x^{-}\right) \\
& =g(|x|)+g(-|x|), \quad \text { since } g \text { is o.a. }
\end{aligned}
$$

LEMMA 5. Let $E$ be a Banach lattice and $f: E \rightarrow E^{*}$ be a nondecreasing continuous gradient map such that (1) the $x$-translates are othongonally additive for all $x$ in $E$, and (2) if $y \perp z, f(x+y) z=f(x) z$ for all $x$ in $E$. Then the translates of the real valued function $F$ on $E$, where $F$ is the function defined by $F(x)=\int_{0}^{1} f(t x) d t$, are orthogonally additive.

ProOF. Let $y, z$ be in $E$ such that $y \perp z$. Now

$$
\begin{aligned}
F(x+y+z)-F(x)= & \int_{0}^{1} f(t(x+y+z))(x+y+z) d t-\int_{0}^{1} f(t x) x d t \\
= & \int_{0}^{1}[f(t(x+y+z))-f(t x)] x d t \\
& +\int_{0}^{1} f(t(x+y+z)) y+f(t(x+y+z)) z d t
\end{aligned}
$$

Since the translates of $f$ are o.a., the first integral on the right-hand side of the last equation above

$$
=\int_{0}^{1}\{f(t(x+y))+f(t(x+z))-2 f(t x)\} x d t,
$$

while the second integral, using the property (2), is verified to be

$$
=\int_{0}^{1}[f(t(x+y)) y+f(t(x+z)) z] d t
$$

as a consequence of the condition (2) in the hypothesis of the lemma. Thus if $y \perp z$, then

$$
\begin{aligned}
F(x+y+z)-F(x) & =\int_{0}^{1} f(t(x+y))(x+y)+f(t(x+z))(x+z)-2 f(t x) x d t \\
& =F(x+y)+F(x+z)-2 F(x)
\end{aligned}
$$

verifying that the $x$-translates of $F$ are o.a., completing the proof of the lemma.

THEOREM 6. Let $f: E \rightarrow E^{*}$ be a nondecreasing gradient mapping. If $F$ is the function as defined in the preceding lemma, then

$$
F(y)+F(-y) \leq F(z)+F(-z) \text { for all } y, z \text { in } E
$$

such that $0 \leq y \leq z$.

ProOF. As a preliminary case let $f(0)=0$. Since $0 \leq y \leq z$ and $f$ is nondecreasing, $0 \leq f(t y) \leq f(t z)$ for $t \geq 0$. Thus $f(t y) y \leq f(t z) z$. On integration w.r.t. $t$ from 0 to 1 , it follows that $F(y) \leq F(z)$. Also since $0 \geq-y \geq-z$, $f(-t z) \leq f(-t y) \leq 0$ for all $t \geq 0$. Hence $f(-t y)(-y) \leq f(-t z)(-z)$. Thus again on integration w.r.t. $t, F(-y) \leq F(-z)$. Hence if $f(0)=0$ the inequality (*) obtains. If $f(0) \neq 0$, consider the map $g: E \rightarrow E^{*}$ defined by $g(x)=f(x)-f(0)$. 
Then $g$ is a nondecreasing continuous gradient mapping, and if $G(\xi)=\int_{0}^{1} g(t \xi) \xi d t$ for all $\xi$ in $E$, then

$$
G(y)+G(-y) \leq G(z)+G(-z) \text { if } 0 \leq y \leq z \text {, since } g(0)=0 .
$$

Now it is verified that

$$
G(\xi)=F(\xi)-f(0) \xi
$$

Since $f(0)$ is a linear functional, it follows from (1) and (2) that

$$
F(y)+F(-y) \leq F(z)+F(-z),
$$

as desired.

The theorem stated below asserts the converse of Theorem 3 .

THEOREM 7. Let $f$ be a continuous nondecreasing gradient mapping on the Banach lattice $E$ into its dual $E^{*}$. Further let (1) the translates of $f$ be o.a., i.e. the functions $f(x+y)$ are o.a. in $y$ for all $x$ in $E$, and (2) for all pairs of orthogonal vectors $y, z$ in $E$ and for all $x$ in $E$ let $f(x+y) z=f(x) z$. If $F$ is the real valued function on $E$, obtained by setting $F(\xi)=\int_{0}^{1} f(t \xi) \xi d t$, then $F$ is symmetrically monotone.

Proof. Let $x \in E$ be fixed. Let $y, z$ be in $E$ such that $|y| \leq|z|$. Consider the $x$-translate of $F$ defined by $G(\xi)=F(x+\xi)-F(x)$. Then $G^{\prime}(\xi)=f(x+\xi)$ for all $\xi$ in $E$. Thus $f(x+\xi)$ is a nondecreasing gradient mapping on $E$. Since $G(0)=0$ and $G^{\prime}(\xi)=f(x+\xi)$, it follows that $G(\xi)=\int_{0}^{1} f(x+t \xi) \xi d t$. Since $|y| \leq|z|$,

$$
G(|y|)+G(-|y|) \leq G(|z|)+G(-|z|)
$$

as inferred from Lemma 6. Since $G$ is o.a. as a consequence of Lemma 5, applying Lemma 4 the inequality (*) above yields $G(y)+G(-y) \leq G(z)+G(-z)$ if $|y| \leq|z|$. Thus if $|y| \leq|z|$,

$$
F(x+y)+F(x-y)-2 F(x) \leq F(x+z)+F(x-z)-2 F(x) .
$$

Hence $F$ is symmetrically monotone.

The next theorem summarizes Theorems 1, 3 and 7.

THEOREM 8. Let $E$ be a Banach lattice and $G$ be a $C^{1}$-real valued function on $E$ with $f$ the gradient map of $G$. Then the following properties of $G$ and $f$ are equivalent:

(1) $G$ is convex and for each $x \in E$, the $x$-translate of $G$ is orthogonally additive.

(2) The map $f: E \rightarrow E^{*}$ satisfies the following conditions:

(a) $f$ is nondecreasing

(b) for each $x \in E$, the translate of $f$ is orthogonally additive, and

(c) if $y, z$ are orthogonal vectors in $E$, then $f(x+y) z=f(x) z$ for all $x$ in $E$.

Proof. (1) $\Rightarrow(2)$. From Theorem 1, it follows that (1) implies $G$ is symmetrically monotone. Thus it is inferred from Theorem 3 that $f$ satisfies all the conditions in (2) above. Next to verify (2) $\Rightarrow(1)$ note that if $F(\xi)=\int_{0}^{1} f(t \xi) \xi d t$, then $F$ is symmetrically monotone as a consequence of Theorem 7. Since $G(\xi)-G(0)=F(\xi)$, $G$ is also symmetrically monotone. Applying Theorem 1 , it follows that $G$ satisfies the properties stated in (1). 
The following examples signify the importance of Theorem 8 .

EXAMPLE 1. If $E$ is a Banach lattice of dimension $\geq 2$, then there is a $C^{1}$ convex real valued function on $E$ such that its gradient fails to be nondecreasing.

The construction of one such function is as follows. If $E$ is a Banach lattice of $\operatorname{dim} \geq 2$, then there exist positive orthogonal vectors $x$ and $y$ in $E$. Let $f_{1}, f_{2}$ be two positive functionals in $E^{*}$ such that $f_{1}(x)=1, f_{1}(y)=0, f_{2}(x)=0$, and $f_{2}(y)=2$. Let $f \in E^{*}$ be the functional defined by $f=f_{1}-f_{2}$. Then if $F(x)=(f(x))^{2}$, it is verified that $F$ is a $C^{1}$-convex real valued function on $E$ with its derivative at $\xi$, $F^{\prime}(\xi)=2 f(\xi) f$, for all $\xi \in E$. It is verified that $F^{\prime}(x)=2 f$, while $F^{\prime}(x+y)=-2 f$. Since $x \leq x+y$, and $2 f$ is not less than $-2 f, F^{\prime}$ is not nondecreasing.

In this example it is noted that $F$ does not satisfy the second part of condition (1) in Theorem 8.

EXAMPLE 2. If $E$ is a Banach lattice of $\operatorname{dim} \geq 2$, then there is a nonconvex $C^{1}$-real valued function $F$ on $E$ with its gradient $F^{\prime}$ satisfying the conditions (i) $F^{\prime}$ is nondecreasing, (ii) the translates of $f^{\prime}$ are orthogonally additive, and (iii) $F^{\prime}$ does not satisfy condition 2 (c) of Theorem 8 .

An example of such a function $F$ is as follows.

If $E$ is a Banach lattice of $\operatorname{dim} \geq 2$, there exist two positive orthogonal vectors $x$ and $y$ in $E$. Let $f_{1}, f_{2}$ be two positive functionals in $E^{*}$ such that $f_{1}(x)=1$, $f_{1}(y)=0 f_{2}(y)=1$, and $f_{2}(x)=0$. Consider the real valued function $F$ on $E$ defined by $F(\xi)=f_{1}(\xi) f_{2}(\xi)$. Since $F((x+y) / 2)$ is not $\leq \frac{1}{2} F(x)+\frac{1}{2} F(y)$ as verified by simple computation, it follows that $F$ is not convex. Further, $F$ is of class $C^{1}$ with the derivative of $F$ at $\xi$ given by $F^{\prime}(\xi)=f_{1}(\xi) f_{2}+f_{2}(\xi) f_{1}$. Since $f_{1}, f_{2}$ are positive functionals it is verified that $F^{\prime}(\xi) \leq F^{\prime}(\eta)$ if $\xi \leq \eta$. Thus $F^{\prime}$ is nondecreasing. Further, since $F^{\prime}: E \rightarrow E^{*}$ is a linear transformation, the translates of $F^{\prime}$ are additive, in particular orthogonally additive. However the function $F^{\prime}$ fails to satisfy condition 2(c) of Theorem 8, i.e. if $p, q, r$ are any three vectors in $E$ with $p \perp r$, then $F^{\prime}(p+q) r \neq F^{\prime}(q) r$ in general, for let $p=x, q=y$, and $r=y$, where $x, y$ are as chosen in the preceding paragraph. From the choice of $f_{1}$ and $f_{2}$, it is verified that $F^{\prime}(x+y) y \neq F^{\prime}(y) y$. Hence the function $F$ thus constructed satisfies all the requirements.

The author is indebted to the referee for several helpful comments.

\section{REFERENCES}

1. M. S. Berger, Nonlinearity and functional analaysis, Academic Press, New York, 1977.

2. H. Cartan, Calcul différentiel dans les espaces de Banach, Hermann, Paris, 1967.

3. L. Drewnowski and W. Orlicz, On representation of orthogonally additive functions, Bull. Acad. Polon. Sci. Sér. Sci. Math. Astronom. Phys. 16 (1968), 883-888.

4. J. Dieudonné, Foundations of modern analysis, Academic Press, New York, 1969.

5. G. H. Hardy, J. E. Littlewood and G. Polya, Inequalities, Cambridge Univ. Press, London, 1934.

6. H. E. Lacey, The isometric theory of classical Banach spaces, Springer-Verlag, New York, Heidelberg and Berlin, 1974.

7. S. Lang, Differentiable manifolds, Addison-Wesley, Reading, Mass., 1972.

8. J. Lindenstrauss and L. Tzafriri, Classical Banach spaces. II, Springer-Verlag, New York, Heidelberg and Berlin, 1979.

9. M. Marcus and V. Mizel, Complete characterization of functions which act, via superpositions on Sobolev spaces, Trans. Amer. Math. Soc. 251 (1979), 187-218. 
10. V. Mizel, Characterization of nonlinear transformations possessing kernels, Canad. J. Math. 22 (1970), 449-471.

11. H. H. Schaefer, Banach lattices and positive operators, Springer-Verlag, New York, Heidelberg and Berlin, 1974.

12. K. Sundaresan, Convex functions on Banach lattices, preprint.

13. K. Sundaresan and S. Swaminathan, Geometry and nonlinear analysis, Lecture Notes in Math., vol. 1131, Springer-Verlag, New York, Heidelberg and Berlin, 1985.

Department of Mathematics, Texas A \& M University, College Station, TEXAS 77843

Department of Mathematics, Cleveland State University, Cleveland, Ohio 44115 (Current address) 